Implementation of the new multichannel X-mode edge density profile reflectometer for the ICRF antenna on ASDEX Upgrade

D. E. Aguiam, A. Silva, V. Bobkov, P. J. Carvalho, P. F. Carvalho, R. Cavazzana, G. D. Conway, O.

D’Arcangelo, L. Fattorini, H. Faugel, A. Fernandes, H. Fünfgelder, B. Gonçalves, L. Guimarais, G. De Masi, L. Meneses, J. M. Noterdaeme, R. C. Pereira, G. Rocchi, J. M. Santos, A. A. Tuccillo, O. Tudisco, and ASDEX Upgrade Team

Citation: Review of Scientific Instruments 87, $11 \mathrm{E722} \mathrm{(2016);} \mathrm{doi:} \mathrm{10.1063/1.4961558}$

View online: http://dx.doi.org/10.1063/1.4961558

View Table of Contents: http://aip.scitation.org/toc/rsi/87/11

Published by the American Institute of Physics

Articles you may be interested in

Multi-channel poloidal correlation reflectometry on experimental advanced superconducting tokamak

Review of Scientific Instruments 87, 11E70711E707 (2016); 10.1063/1.4960162

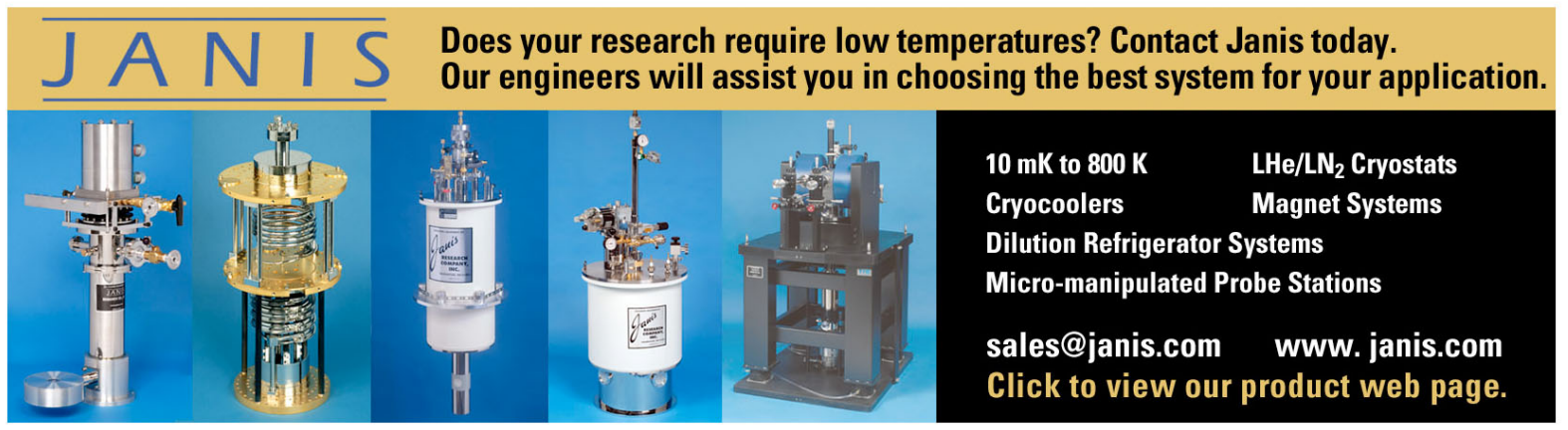




\title{
Implementation of the new multichannel X-mode edge density profile reflectometer for the ICRF antenna on ASDEX Upgrade
}

\author{
D. E. Aguiam, ${ }^{1, a)}$ A. Silva, ${ }^{1}$ V. Bobkov,${ }^{2}$ P. J. Carvalho, ${ }^{1}$ P. F. Carvalho, ${ }^{1}$ R. Cavazzana,${ }^{3}$ \\ G. D. Conway, ${ }^{2}$ O. D'Arcangelo, ${ }^{4}$ L. Fattorini, ${ }^{5} \mathrm{H}$. Faugel, ${ }^{2}$ A. Fernandes, ${ }^{1}$ H. Fünfgelder, ${ }^{2}$ \\ B. Gonçalves, ${ }^{1}$ L. Guimarais, ${ }^{1}$ G. De Masi ${ }^{3}$ L. Meneses, ${ }^{1}$ J. M. Noterdaeme,${ }^{2,6}$ \\ R. C. Pereira, ${ }^{1}$ G. Rocchi, ${ }^{4}$ J. M. Santos,${ }^{1}$ A. A. Tuccillo, ${ }^{4}$ O. Tudisco, ${ }^{4}$ \\ and ASDEX Upgrade Team ${ }^{2, b)}$ \\ ${ }^{1}$ Instituto de Plasmas e Fusão Nuclear, Instituto Superior Técnico, Universidade de Lisboa, \\ 1049-001 Lisboa, Portugal \\ ${ }^{2}$ Max-Planck-Institut für Plasmaphysik, Boltzmannstr. 2, D-85748 Garching, Germany \\ ${ }^{3}$ Consorzio RFX (CNR, ENEA, INFN, Universitá di Padova, Acciaierie Venete SpA), Corso Stati Uniti 4, \\ 35127 Padova, Italy \\ ${ }^{4}$ ENEA, Dipartimento FSN, C. R. Frascati, Via E. Fermi 45, 00044 Frascati, Roma, Italy \\ ${ }^{5}$ Universitá degli Studi Milano Bicocca, Dipartimento di Fisica, Piazza della Scienza 3, 20126 Milano, Italy \\ ${ }^{6}$ Applied Physics Department, Ghent University, B-9000 Gent, Belgium
}

(Presented 9 June 2016; received 6 June 2016; accepted 28 July 2016;

published online 29 August 2016)

\begin{abstract}
A new multichannel frequency modulated continuous-wave reflectometry diagnostic has been successfully installed and commissioned on ASDEX Upgrade to measure the plasma edge electron density profile evolution in front of the Ion Cyclotron Range of Frequencies (ICRF) antenna. The design of the new three-strap ICRF antenna integrates ten pairs (sending and receiving) of microwave reflectometry antennas. The multichannel reflectometer can use three of these to measure the edge electron density profiles up to $2 \times 10^{19} \mathrm{~m}^{-3}$, at different poloidal locations, allowing the direct study of the local plasma layers in front of the ICRF antenna. ICRF power coupling, operational effects, and poloidal variations of the plasma density profile can be consistently studied for the first time. In this work the diagnostic hardware architecture is described and the obtained density profile measurements were used to track outer radial plasma position and plasma shape. [http://dx.doi.org/10.1063/1.4961558]
\end{abstract}

\section{INTRODUCTION}

The operation and coupling of Ion Cyclotron Range of Frequencies (ICRF) power to the plasma are greatly influenced by the dynamic behaviour of the plasma layers in front of the antenna. Simulations have shown that the induced RF sheaths contribute to the generation of $\mathrm{E} \times \mathrm{B}$ convective transport, impurity generation, hot spots, and power dissipation. ${ }^{1}$ Even though indirect measurements, such as measuring density and temperature along the toroidal magnetic field lines during ICRF operation, corroborated the simulation results, no direct experimental observation of these effects was available. A new three-strap ICRF antenna was designed for ASDEX Upgrade (AUG) with the aim of reducing wall $\mathrm{W}$ impurity sputtering ${ }^{2}$ in a collaboration between IPP, ASIPP, and ENEA. This new antenna design also integrates ten small microwave reflectometry antenna pairs (Fig. 1), which look at the plasma from different toroidal and poloidal locations. ${ }^{3}$

The new multichannel density profile reflectometry system was designed to use the available embedded antennas to study the evolution of the edge electron density profile at

\footnotetext{
Note: Contributed paper, published as part of the Proceedings of the 21st Topical Conference on High-Temperature Plasma Diagnostics, Madison, Wisconsin, USA, June 2016.

a)daguiam@ipfn.tecnico.ulisboa.pt

b) See authors list of A. Kallenbach et al., Nucl. Fusion 51, 094012 (2011).
}

three different poloidal locations directly in front of the ICRF antenna. The new three-strap ICRF antenna and embedded reflectometry diagnostic were installed successfully on AUG in 2015. This project was an international collaboration between the ASDEX Upgrade, Germany, ENEA, Italy and Instituto Superior Técnico, Portugal.

\section{REFLECTOMETRY DIAGNOSTIC}

The implemented multichannel reflectometry diagnostic was designed to measure edge plasma electron density profiles up to $2 \times 10^{19} \mathrm{~m}^{-3}$ in X-mode in the typical $1.5 \mathrm{~T}-2.7 \mathrm{~T}$ magnetic fields of AUG. The probing wave frequency is swept in the extended U-band between $40 \mathrm{GHz}$ and $68 \mathrm{GHz}$. This diagnostic uses a modular design, in which the microwave hardware is attached to the reflectometry antenna waveguides, near the tokamak vessel, while the control and acquisition systems, connected to the AUG network, are placed inside the torus hall but kept away from the magnetic interference during the discharge.

The system integrates a universal time-to-digital converter ${ }^{4}$ to generate synchronized triggers for the acquisition and frequency sweep generation. The acquisition system uses the Advanced Telecommunications Computing Architecture standard and communicates with the host computer through a PCIExpress link. There are 8 acquisition channels with a 13-bit 


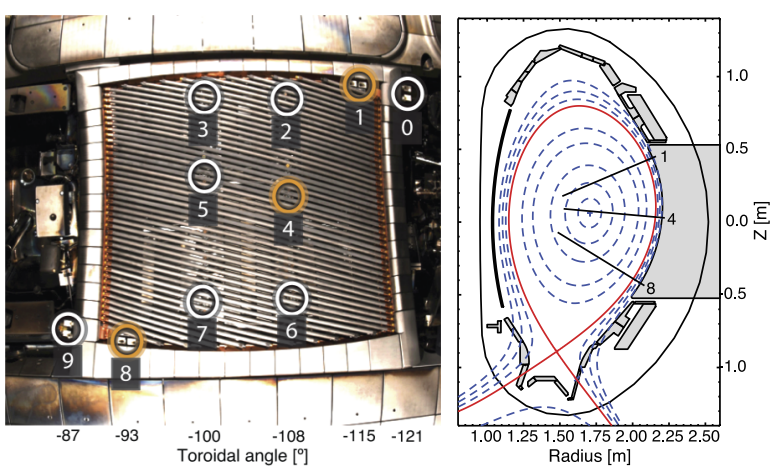

FIG. 1. (Left) ICRF antenna embedded reflectometry antenna pairs locations. (Right) Poloidal cross section of vessel and lines of sight of connected reflectometry antennas 1,4 , and 8 .

resolution, a maximum sampling rate of $200 \mathrm{MSamples} / \mathrm{s}$, and a total of $512 \mathrm{MB}$ memory per channel. ${ }^{5}$ Each reflectometer channel uses two (I and Q) acquisition channels.

In a typical reflectometer operational configuration, the full frequency range is swept in $15 \mu \mathrm{s}$, generating an electron density profile every $100 \mu \mathrm{s}$. This configuration allows covering $8.9 \mathrm{~s}$ of the AUG discharge, limited by the data acquisition system memory. In fast sampling configuration, a density profile can be obtained every $25 \mu$ s covering around $2.2 \mathrm{~s}$, useful for detailed sampling of fast plasma events.

A total of 10 reflectometry antenna pairs, consisting of transmitting and receiving antennas in bistatic configuration, are embedded in the ICRF antenna \#4, in sector 12 of the tokamak (see Fig. 1). The outer antennas $(0,1,8$, and 9) are pyramidal horn antennas located between the last Faraday straps and the ICRF antenna limiter. The central antennas (2-7) use smaller WR42 waveguide tapers to look through the small gap between the Faraday shield straps. The reflectometry antennas are polarized for $\mathrm{X}$-mode propagation and are aligned with the Faraday straps, which match the average magnetic field pitch angle of AUG discharges. A different magnetic pitch angle results in a lower amplitude of the $\mathrm{X}$-mode component resulting only in a decrease of the received reflection power. However, this power is still in the measurable range and does not affect the group delay measurement for profile inversion. The CO12 port vacuum flange allowed the access of only 14 WR 19 waveguides, restricting access to only 7 preselected reflectometry antenna pairs at a time without rerouting the in-vessel waveguides. The implemented diagnostic uses three channels, connected to antennas 1,4 , and 8 , with the possibility for new channels in future upgrades.

\section{A. Reflectometer architecture and operation}

In density profile reflectometry, the probing wave propagates in the plasma and is reflected at a cut-off layer where the index of refraction goes to zero, depending on the probing wave frequency and the local plasma characteristics. By sweeping the probing frequency it is possible to estimate the electron density of the plasma layer at which the wave is reflected. Mixing the reflected wave with a local oscillator signal results in a beat signal whose frequency is proportional to the group delay of the probing wave propagating in the plasma. Density profiles can be measured from almost zero

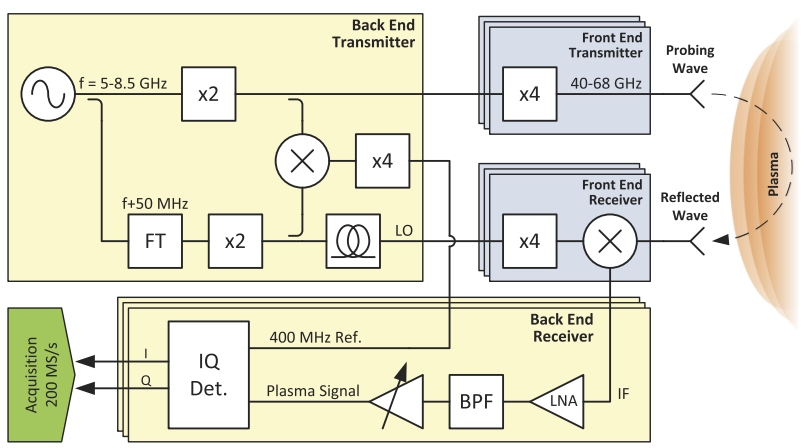

FIG. 2. Heterodyne topology of the reflectometer.

density using the $\mathrm{X}$-mode upper cut-off, up to a maximum density limited by the plasma magnetic field and the quality of the signal near the end of the probing band. This diagnostic uses the full coherent heterodyne architecture (see Fig. 2) that provides in-phase and quadrature (IQ) beat signals for each of the reflectometer channels.

A single Voltage Controlled Oscillator (VCO) is used to generate a 5-8.5 GHz signal, which is split into two branches. In the transmitting branch, this signal is doubled, power split in three, and used as the source of each transmitter frontend. Each transmitter front-end consists of an active frequency quadrupler attached directly to the reflectometry transmitting antenna waveguide, providing the $40-68 \mathrm{GHz}$ probing signal with a maximum power output of $+16 \mathrm{dBm}$. In the local oscillator (LO) branch, a frequency translator module introduces a $50 \mathrm{MHz}$ shift in the source signal to generate a coherent 5.05-8.55 GHz reference signal, which is doubled and quadrupled to drive the mixers LO port.

The receiver front-end consists of an active frequency quadrupler and a fundamental mixer. The reflected wave is mixed with the LO signal generating a beat signal, whose frequency is proportional to the delay of the reflected wave, centered at an intermediate frequency of $400 \mathrm{MHz}$. A coaxial delay line partially compensates the delay introduced by the long waveguide transmission line (about $10 \mathrm{~m}$ ), and keeps the beat frequency within the IQ detector bandwidth $(100 \mathrm{MHz})$. In the receiver back-end, the beat signal is filtered and amplified. The quadrature detector uses the synchronized reference to demodulate the beat signal into its IQ components, which are then acquired.

The raw IQ signals are then calibrated to remove the contribution of the propagation in the waveguide to the group delay. A spectral analysis of the signal is performed to determine the start of the plasma reflection signal, which corresponds to the zero density reflection, known as the first fringe (FF), and can occur anywhere in the $40-68 \mathrm{GHz}$ band, depending on the local magnetic field and the distance of the plasma to the antenna. The measured group delay, the probing wave frequency, and the magnetic field profile along the line of sight obtained from the equilibrium codes are then used to invert the density profile. ${ }^{6}$

\section{EXPERIMENTAL RESULTS}

In order to validate our new reflectometry diagnostic data, the measured density profiles were compared with other 


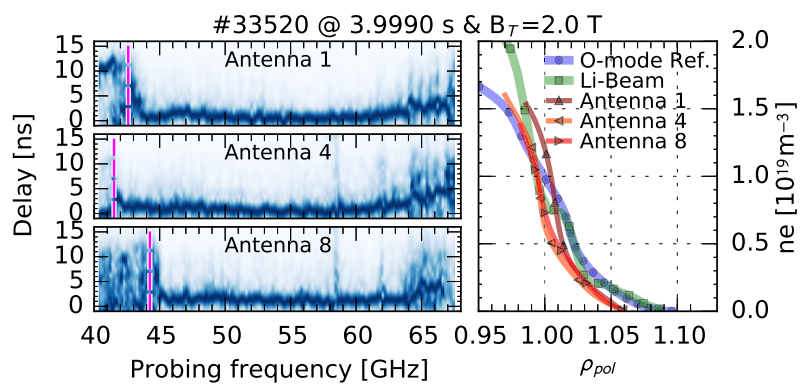

FIG. 3. (Left) Measured reflectometry antenna signal delay and estimated first fringe frequency (dashed line). (Right) Comparison of measured density profiles with other diagnostic data.

available data from the lithium beam and O-mode reflectometry diagnostics installed on AUG. For this purpose a stable plasma with no ICRF heating was chosen and the measured group delay spectra, used to calculate the density profiles of each reflectometry channel, are represented in Fig. 3 (left). Each FF frequency (dashed lines) depends on the local magnetic field in front of the antenna, and the plasma signal region size, from FF up to the end of the probing band, limits the maximum measurable density. The corresponding density profiles are shown in Fig. 3 (right), normalized to the $\rho_{\text {pol }}$ flux coordinates. A good overlapping of the measured profiles with the ones from other diagnostics can be observed, corroborating the new diagnostic data.

A commissioning shot was designed to study the performance of the new reflectometry diagnostic in tracking the density profiles modification during variations of the plasma outer radial position and plasma shape, by changing plasma triangularity, and toggling ICRF heating, as shown in Fig. 4. The density profile contour lines measured in front of each antenna were mapped to the major radius with a height equal to the magnetic axis. It can be observed that these measured

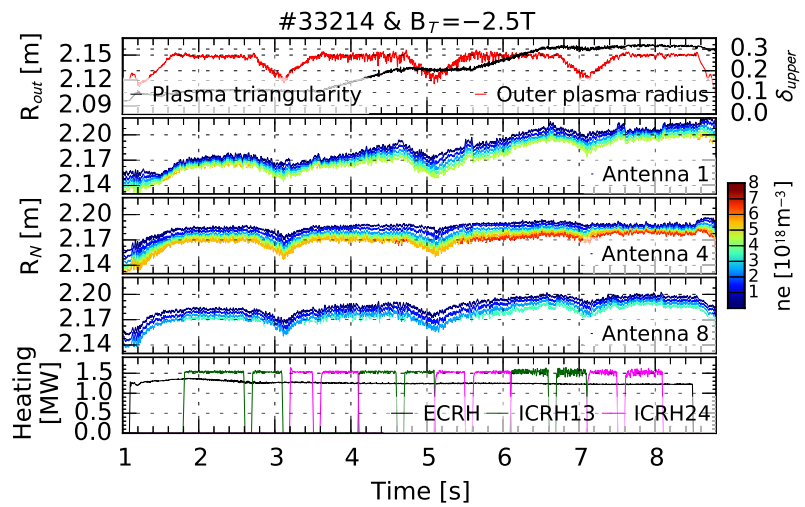

FIG. 4. Evolution of the measured density profile contour lines during a scan of the plasma outer radial position and triangularity. profiles agree with the variation of the plasma radial position. The variation in plasma triangularity introduces a poloidal difference in plasma shape, which can be observed in the profiles measured in the upper (1) and lower (8) antennas. The measured radial displacement of the edge isodensity layers in front of each connected antenna, represented in Fig. 4, agrees with the separatrix displacement. In addition, it can also be noticed that ICRF operation results in the contraction of the edge density profile measured in the upper antenna (1). This indicates an interesting result that poloidal differences may be studied by comparing different antenna measurements.

\section{SUMMARY AND CONCLUSIONS}

The new multichannel X-mode edge plasma electron density profile reflectometry diagnostic for the ICRF antenna has been successfully installed and commissioned on ASDEX Upgrade, Germany. Changes in the plasma outer radial position and plasma triangularity were observed using the density profiles obtained in front of each antenna location. Early results show that this multichannel reflectometry diagnostic will enhance and improve the analysis of plasma poloidal variations and the observation of convective cells during ICRF operation.

\section{ACKNOWLEDGMENTS}

This work has been carried out within the framework of the EUROfusion Consortium and has received funding from the Euratom research and training programme 2014-2018 under Grant Agreement No. 633053. IST activities also received financial support from Fundação para a Ciência e Tecnologia through Project No. UID/FIS/50010/2013 and the Ph.D. Scholarship Grant No. FCT-SFRH/BD/52414/2013. The views and opinions expressed herein do not necessarily reflect those of the European Commission.

${ }^{1}$ D. A. D'Ippolito and J. R. Myra, Phys. Plasmas 13, 102508 (2006).

${ }^{2}$ V. Bobkov, M. Balden, R. Bilato, F. Braun, R. Dux, A. Herrmann, H. Faugel, H. Fünfgelder, L. Giannone, A. Kallenbach, H. Maier, H. Müller, R. Neu, J.-M. Noterdaeme, T. Pütterich, V. Rohde, N. Tsujii, F. Zeus, and H. Zohm, Nucl. Fusion 53, 093018 (2013).

${ }^{3}$ O. Tudisco, A. Silva, S. Ceccuzzi, O. D'Arcangelo, G. Rocchi, H. Fuenfgelder, V. Bobkov, R. Cavazzana, G. D. Conway, J. Friesen, B. Gonçalves, A. Mancini, L. Meneses, J. M. Noterdaeme, G. Siegl, A. Simonetto, N. Tsujii, A. A. Tuccillo, T. Vierle, I. Zammuto, ASDEX Upgrade Team, and FTU Team, AIP Conf. Proc. 1580, 566 (2014).

${ }^{4}$ G. Raupp, R. Cole, K. Behler, M. Fitzek, P. Heimann, A. Lohs, K. Lüddecke, G. Neu, J. Schacht, W. Treutterer, D. Zasche, T. Zehetbauer, and M. Zilker, Fusion Eng. Des. 66-68, 947 (2003).

${ }^{5}$ R. C. Pereira, A. M. Fernandes, A. C. Neto, J. Sousa, A. J. Batista, B. B. Carvalho, C. M. B. A. Correia, and C. A. F. Varandas, IEEE Trans. Nucl. Sci. 57, 683 (2010).

${ }^{6}$ E. Mazzucato, Rev. Sci. Instrum. 69, 2201 (1998). 